\title{
CONF- $9611.10--16$ \\ THE UPGRADE OF THE BROOKHAVEN LINAC ISOTOPE PRODUCER (BLIP) AND THE BNL LINAC
}

\author{
L. F. Mausner and J. G. Alessi \\ Medical Department and Alternating Gradient Synchrotron Department
}

Brookhoven National Laboratory, Upton, New York 11973

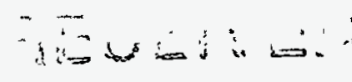

DEC 231996

\begin{abstract}
An upgrade project was recently completed on the $200 \mathrm{MeV} \mathrm{H}^{-}$linac and the Brookhaven Linac Isotope
Producer (BLIP) in order to improve radioisotope production capacity and reliability. The average beam current has increased from $60 \mu \mathrm{A}$ to $150 \mu \mathrm{A}$. The increased average current is the result of increases in peak current, from $25 \mathrm{~mA}$ to $37 \mathrm{~mA}$, pulse repetition rate, from 5 to $7.5 \mathrm{~Hz}$, and pulse width, from 500 to $530 \mu \mathrm{s}$. To achieve this performance the $35 \mathrm{keV}, 750 \mathrm{keV}$ and $200 \mathrm{MeV}$ beam transport were improved, the RF transmission lines and RF power supplies replaced. Improvements to the linac control system, and the optics and vacuum system of the $200 \mathrm{MeV}$ transport were implemented. At BLIP the target cooling system was upgraded to $35 \mathrm{~kW}$ and automated, the targets, and target mechanical systems replaced with a more robust design, and the control system upgraded. With these enhancements BLIP is ready to address the lack of availability of accelerator produced medical and research isotopes.
\end{abstract}

\section{INTRODUCTION}

The BLIP, built in 1972, utilizes the excess beam capacity of the $200 \mathrm{MeV}$ proton linac injector for the Alternating Gradient Synchrotron to serve as national resource for the production of many isotopes which are crucial to nuclear medicine for both research and routine clinical use in patients and which are generally unavailable elsewhere. The radionuclides presently producible at BLIP are ${ }^{7} \mathrm{Be}$, ${ }^{28} \mathrm{Mg},{ }^{52} \mathrm{Fe},{ }^{65} \mathrm{Zn},{ }^{67} \mathrm{Cu},{ }^{68} \mathrm{Ge},{ }^{82} \mathrm{Sr},{ }^{83} \mathrm{Rb},{ }^{95 \mathrm{~m}, 96} \mathrm{Tc}$ and ${ }^{97} \mathrm{Ru}$. In order to respond to a mounting national need for a continuous and reliable source of isotopes, we have upgraded the BLIP and linac. Table 1 shows the linac performance before and after the upgrade.

\section{LINAC MODIFICATIONS 35 keV Beam Transport Line}

The beam from the magnetron surface-plasma $\mathrm{H}^{-}$source is matched in to the RFQ by two pulsed magnetic solenoid lenses. In order to improve matching of the beam into the RFQ, this line was shortened by $0.7 \mathrm{~m}$, to $1.4 \mathrm{~m}$, by removing a fast chopper box which was no longer used. In addition, calculations showed that further improvements in matching would result if the first solenoid were moved

TABLE 1. Linac Performance

$\mathrm{H}^{*}$ beam current Repetition rate

Beam width

Average current

\begin{tabular}{c} 
Before Upgrade \\
\hline $25 \mathrm{~mA}$ \\
$5 \mathrm{~Hz}$ \\
$500 \mu \mathrm{s}$ \\
$62 \mu \mathrm{s}$
\end{tabular}

After Upgrade

$37 \mathrm{~mA}$

$7.5 \mathrm{~Hz}$

$530 \mu \mathrm{s}$

$146 \mu \mathrm{s}$ closer to the source, and the second solenoid closer to the RFQ. With these changes, the transmission through the RFQ improved from the $70-80 \%$ range to $80-90 \%$, depending on source operating conditions. The emittance of the beam in front of the RFQ was reduced by about $-20 \%$. Typical current out of the RFQ is now $65 \mathrm{~mA}$, and the maximum current through the RFQ is $80 \mathrm{~mA}, 82 \%$ transmission.

\section{$750 \mathrm{keV}$ Beam Transport Line}

There is a $6 \mathrm{~m}$ transport line from the RFQ to linac, to accommodate a pulsed dipole where polarized $\mathrm{H}^{-}$comes from a second beamline, and a fast beam chopper. There are three bunchers and 13 quadrupoles in this line. Transmission in this line was only $-75 \%$, with losses early in the line caused by the fact that the first quadruple after the RFQ was not close enough to catch the beam before it got too large. In order to reduce the beam divergence quickly coming out of the RFQ, a $1.1 \mathrm{~cm}$ aperture, $3.5 \mathrm{~cm}$ long permanent magnet quadruple was placed in the end flange of the RFQ, only $2.1 \mathrm{~cm}$ from the RFQ vane tip. In addition, this quadruple can be moved transversely while running beam, via micrometer adjustment outside vacuum, in order to steer the beam.

Additional changes in the line were to move the first quadruple triplet $9 \mathrm{~cm}$ closer to the RFQ, and to convert a final quadruplet (which had been running as a triplet), into a real triplet. We also removed all magnets from the line and had the fields precisely measured. All magnets were carefully surveyed when reinstalled, particularly trying to eliminate quadruple rotations, which leads to emittance 
growth of the beam. With these improvements, the transmission from the RFQ to linac was improved to $\sim 85 \%$. We believe that the remaining loss comes from the 12 "grids" (made of thin tungsten strips) in the line, in the three buncher cavities.

With this $50 \%$ higher current at the linac entrance, the linac transmission remained the same, with $-70 \%$ being captured in the first tank. This agrees with calculations of the line, and comes from the fact that the line is too long to match longitudinally with only three bunchers. The calculations show that with a fourth buncher the capture into the linac could be $>95 \%$.

\section{$200 \mathrm{MeV}$ Beam Transport Line}

There were both vacuum-related and beam optics improvements in the transport line between the end of the linac and the BLIP targets. Most of the vacuum components in the line were replaced. O-rings were eliminated, and the line now uses all conflat flanges. Aluminum pipe was used in much of the line to minimize activation. Pumping of the line was increased, with new turbo pumps and ion pumps. Apertures in the line were increased wherever possible, and most of the line has either 15.2 or $20.3 \mathrm{~cm}$ diameter beam pipe. Also, two stepping wire profile monitors were modified to give beam profiles in 3 projections (horizontal, vertical, and at $45^{\circ}$. With this, we are able to get 3-dimensional tomographic reconstructions of the beam distribution(1).

There are two bends between the linac and BLIP target, and a quadruple was added to make these bends achromatic.

A stripline position monitor was added between the bends to the BLIP target, at a high dispersion point, to allow monitoring of the linac energy. Further downstream, two octupoles and two quadrupoles were added in order to produce a uniform current density on the BIRC targets, to prevent meiting of some target materials at this increased beam power. This beam flattening exploits the aberrations introduced by the nonlinearity in octupole focussing. We have been able to produce flattened beam profiles on an upstream profile monitor, with good agreement with calculations. Progress on producing a flat distribution on the BLIP target has been slow, due to the long turnaround time ( -1 day) of profile measurements at the target location (via counting of activated foils).

\section{High Power Transmission Line}

In order to improve reliability at the increased linac duty factor, all the linac $30.5 \mathrm{~cm}$ coaxial transmission line was replaced. Up to $6 \mathrm{MW}$ peak power is fed from each of the nine rf systems, through a $3 \mathrm{db}$ power split, and into two ports on each of the nine accelerating cavities, a total of over $200 \mathrm{~m}$ of transmission line for the full linac. Our 25 year old system had disadvantages of having aluminum inner conductor, spring ring if contacts, and was unpressurized. The new transmission line system was and installed by Dielectric Corporation. It has a copper inner conductor and an aluminum alloy outer conductor, is pressurized to 15 psi with dry air, and the connectors for the center conductor are EIA-type finger contacts. We replaced the full system, including the $3 \mathrm{db}$ power splitters, waster loads, breakaway and telescoping sections, and reflectometers. A hybrid phase shifter (mechanically variable) was replaced with a transmission line section of optimum length in each system. The new system has operated very reliably, with low insertion loss, very low probability of voltage breakdown, and improved Sparameters.

\section{Linac RF Power Supplies 7835 Anode Power Supply}

The $6 \mathrm{MW}$ power amplifiers for the linac use Burle 7835 triodes. At the increased current and duty factor of the linac, some of the $60 \mathrm{kV}, 2 \mathrm{~A}, 7835$ anode power supplies would be running at their $2 \mathrm{~A}$ limit, a concern for reliability. In addition, these power supplies, constructed in 1968, are oil filled units that are outside the linac building, connected to capacitor banks by a long high voltage transmission system. It was felt that with the present technology, a dry type power supply could be built and installed in the linac right next to the power amplifier, and in order to meet any future requirements, we settled on a $50 \mathrm{kV}, 5 \mathrm{~A}$ supply. We selected a conventional 6 pulse primary phase controlled dry type transformer rectifier (TR) set, built by Universal Voltronics (UVC). To save money, the power supplies were housed in existing cabinets that formerly housed the charge control amplifiers. These cabinets are fully compatible with the existing lifting fixtures, building crane, and floor space requirements. Seven power supplies have been delivered to BNL and tested, with the remainder due shortly. They will go online in January ' 97.

\section{PLC Controls for RF Systems}

The linac is made up of 9 identical rf stations. Each station has several subsystems, including the driver, 7835 filament supply and cavity, $50 \mathrm{kV}$ supply, capacitor bank, modulator, and local control station (LCS). Each of the subsystems has individual control buckets for $A C$ and high voltage logic. These buckets were built in 1968 around 7400 TTL series components. Replacing these control buckets with more modern components is a necessity because many of the components are no longer available.

We are beginning to implement a new control system, utilizing programmable logical controllers (PLC's). It is designed for fully independent operation of each if system, flexibility and reliability. An Allen Bradley $5 / 40$ processor was chosen for each LCS. A 5/50 ethernet processor was selected for the control room. Each of the 5/40's can scan the subsystems of a system (scanner mode), or be scanned 
by the host in the control room (adaptor mode). There is one network responsible for the data collection and control of each station. To minimize the wiring, each of the subsystems has a miniature processor (Allen Bradley flex IO) that multiplexes the data at 230 kbaud for the $5 / 40$. A single twisted pair links all of the subsystems of a mod together. The subsystems are connected together via the Allen Bradley Remote I/O network. Two other networks are links between each of the systems. The DH+ network runs at $57 \mathrm{kbaud}$ and is responsible for the remote monitoring and control of all 9 systems. The Allen Bradley graphical interface program, Control View, is used to control the supplies. An additional remote I/O network allows the $5 / 40 \mathrm{E}$ in the control room to monitor each mod at $230 \mathrm{kbaud}$ for fast global control.

\section{Linac Controls}

The original 25-year-old linac control system was replaced with a modern modular system fully integrated into the existing AGS distributed control system. Unix workstations provide the operator interface, and are networked using ethernet to front-end computers which are implemented using VMEbus components. A front-end computer located in the Linac Control Room sources four high speed serial communication links using the Datacon field bus, a long-standing BNL standard. Although an old system, Datacon is extremely robust and noise immune, can operate over $2000 \mathrm{ft}$. of coaxial cable, and is relatively inexpensive. Each Datacon link can address up to 256 devices, delivering a 24-bit command and accepting a 32-bit reply. All devices are accessed for each Linac pulse $(7.5 \mathrm{~Hz})$, and in particular, device set points are rewritten for each pulse; thus any sequence of different Linac clients (Booster, BLIP) requiring possibly different settings can be accommodated - a feature termed pulse-to-pulse modulation (PPM). The individual devices are interfaced to the Datacon link via dual-channel cards housed in crates at 11 locations along the Linac. We are controlling and/or monitoring over 400 devices.

\section{BLIP MODIFICATIONS}

\section{Shielding}

The design and operation of this facility has been described previously (2). The higher beam intensity from the Linac creates significantly more radioactivity. Several shielding enhancements were required for personnel safety. The main concern is the very penetrating high energy neutrons created by proton spallation reactions in our targets and structural supports. The arrangement of the BLIP target area is shown schematically in Fig. 1. We modeled the transport of both high and low energy neutrons with the LAHET code system (3). This Monte Carlo code contains two major parts; an intranuclear cascade code for evaluations above $20 \mathrm{MeV}$, and a transport code for
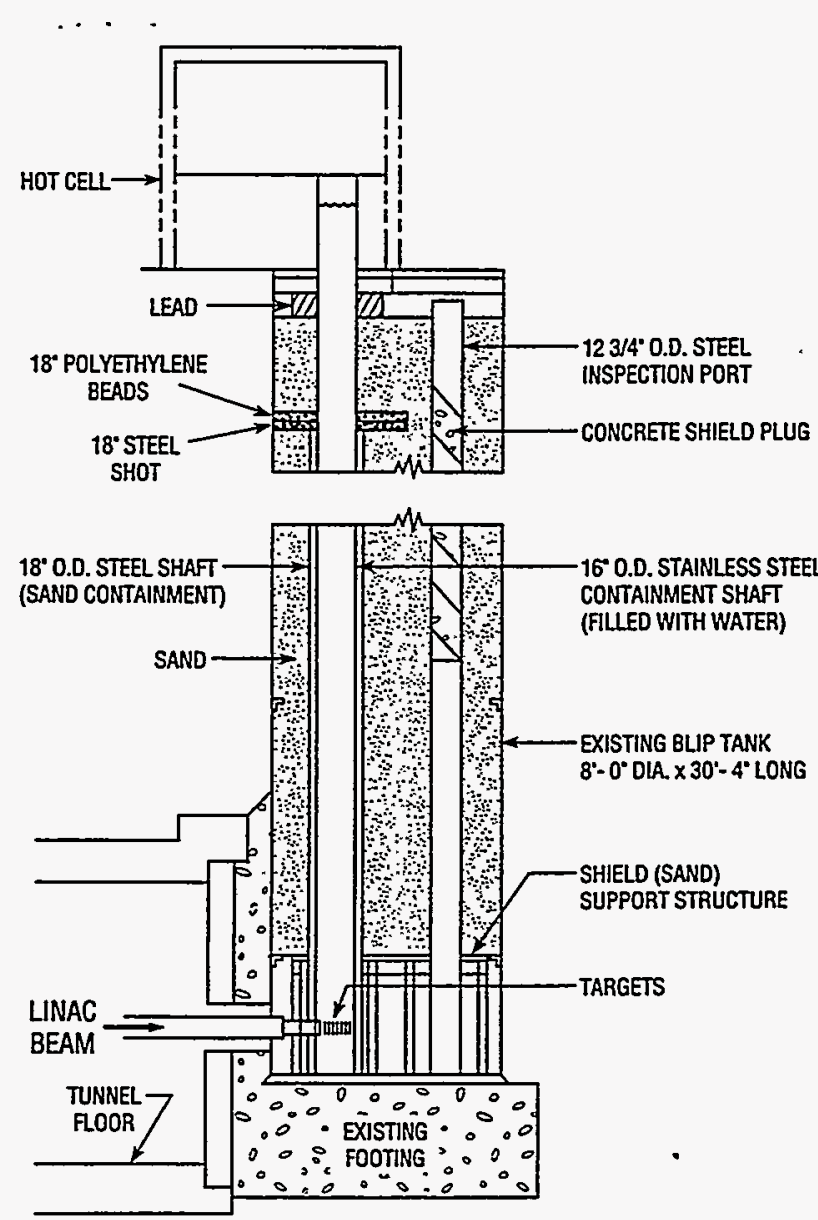

FIGURE 1. Schematic of BLIP target area

calculation from $20 \mathrm{MeV}$ down to thermal energies. Because of the size and complexity of the shielding geometry ( assorted layers of steel, sand, water, concrete etc.) quantitative calculation of neutron doses was not attempted. Rather, relative comparisons of different shielding configurations were performed. This analysis caused us to replace $1 \mathrm{~m}$ of sand shielding with $0.5 \mathrm{~m}$ of steel shot, to attenuate higher energy neutrons, followed by $0.5 \mathrm{~m}$ of polyethylene beads for low energy neutrons, placed around the target shaft at the top of the secondary shaft (approximately $6 \mathrm{~m}$ from the targets).

Shielding in the target handling hot cell was analyzed at the projected increased radionuclide levels with the commercially available code Microshield (Grove Engineering). In response it was necessary to increase the lead glass shielding in the hot cell for target handling from 30 to $36 \mathrm{~cm}$ thickness.

\section{Targets and Cooling System}

At the increased beam intensity new target designs are required. The heat deposited in targets by the proton beam (increasing from $12 \mathrm{~kW}$ to $30 \mathrm{~kW}$ ) is removed by 
conduction and radiation from the target material to the cladding surfaces, and then transferred to the coolant by forced convection. To study target thermal properties we theoretically modeled the mechanical details and steady state heat transfer properties of the targets with a finite element code called ANSYS (Swanson Industries). Initially, we attempted to model the heat transfer properties of three existing BLIP targets; $\mathrm{Zn}$ powder for ${ }^{67} \mathrm{Cu}$ production, $\mathrm{RbCl}$ disks for ${ }^{82} \mathrm{Sr}$, and molten $\mathrm{Ga}$ for ${ }^{68} \mathrm{Ge}$. Qualitative agreement with the previous operating conditions was achieved. However, at the enhanced beam intensity the calculations pointed to central target temperatures for $\mathrm{Zn}$ and $\mathrm{RbCl}$ high enough to cause target destruction.

We investigated alternatives to reduce these extrapolated very high central target temperatures. These were to: a) reduce target thickness, b) change target molecular form, c) embed heat conductors in the target salts, d) scan or wobble the beam spot to reduce power density, or alternatively move the target with respect to the beam, e) flatten the Gaussian beam intensity profile, f) tilt targets with respect to the beam axis. There were trade-offs to each of these alternatives. Reducing target thickness is straightforward, but reduces product yield. A changed molecular form of the target is not always possible due to corrosion, solubility or decomposition problems. Embedded heat conductors increase the target complexity and fabrication cost, as well as create the possibility of target warping at high temperature. Pulse to pulse scanning the beam was rejected because the tighter spot required might cause melting in a single pulse. Continuously scanning the target was rejected because of anticipated mechanical complexity and limited durability. Flattening the beam profile requires new beam optical elements. Finally, tilting the targets significantly increases target drive system size and complexity , as well as reduces radioactivity concentration. We have decided to combine reduced target thickness with a flat profile beam spot and have changed the $\mathrm{Zn}$ metal powder target to a pressed pellet of the much higher melting $\mathrm{ZnO}$.

We also changed the design of the target cooling manifold and target holders. Each stainless steel target holder is attached to a bar which is motor driven vertically with a chain and sprocket system. The original target holder design had $2 \mathrm{~cm}$ inlet and outlet cooling tubes at the bottom of each holder. This led to a circular, swirling water flow across the target faces. The cooling tubes mated with sockets in a fixed manifold at the beam level. These tubes also served to position the target holder in beam. We have replaced this design with a new configuration in which the bottom of the target holder is an open mesh , and a skirt extending below the target holder slides over a rectangular socket to make the water connection. This design allows improved water flow velocity past the target faces with reduced pressure drops, eliminates possible low flow spots on the target faces, and improves mechanical durability. Finally, the target cooling system was replaced with a larger $35 \mathrm{KW}$ system. This is now a closed, dual loop water system which is computer controlled to maintain safe target process water temperatures.

\section{Control System}

BLIP is designed to operate automatically on a 24 hour per day basis, seven days per week, without operator intervention. Operations personnel visit the facility to load or unload targets, maintenance and system development. A control computer provides continuous surveillance of system sensors. Our obsolete system needed to be replaced. The new computer is a standard Pentium class PC, with data acquisition and other input/output interfaces from National Instruments. The process control software package is Labview, also from National Instruments. The software graphically represents the status and parameters of devices in the BLIP and provides graphical front panel user interfaces for operators to monitor, control or test equipment. A detected malfunction can trigger several different alarm outputs, depending on the type and severity of the malfunction. The interlock systems which relate to personnel safety are all hard wired relays, and do not rely on the computer control system. An additional room was constructed to house all the control electronics separate from the radiological areas of the facility. Extra space for storage and contamination monitoring was also constructed.

\section{CONCLUSIONS}

With improvements in beam transport through the 35 and $750 \mathrm{keV}$ sections the average current delivered to BLIP has reached our goal of $146 \mu \mathrm{A}$, and still higher currents should be possible. The upgraded cooling system, and improved target designs at BLIP have safely handled this beam. The many other enhancements to the linac and BLIP should also improve long term reliability as well.

\section{ACKNOWLEDGEMENTS}

We would like to thank the entire BLIP and Linac staffs, the AGS mechanical design, controls, vacuum, and power supply groups, and the RHIC magnet group for their diligent efforts on this project. This work was supported by the U.S. Department of Energy Project 94-E-355 under contract \#DE-AC02-76CH00016.

\section{REFERENCES}

1. Gordon, R., Herman, G. T., "Three-Dimensional Reconstruction from Projections: A Review of Algorithms", International Review of Cytology, 38, 111, (1974).

2. Mausner, L. F., Mirzadeh, S., Schnakenberg H., Srivastava, S. C. "The design and operation of the upgraded BLIP facility for radionuclide research and production"Appl. Radiat. Isot. 41, 367-3748, 1990.

3. Prael, R. E. Lichtenstein, H. , "User guide to LCS: The LAHET code system" LA-UR-89-3014, 1989. 